\title{
Geometrical analysis for assessing torsional alignment of humerus
}

Yo-Lun Chu' ${ }^{1,2,3}$, Cheng-Kuang Chen ${ }^{3}$, Yu-Chia Liư ${ }^{3}$, Tung-Wu Lu' ${ }^{1}$ and Chen-Kun Liaw $4,5,6^{*}$

\begin{abstract}
Background: Compared to other types of surgeries, minimally invasive surgeries (MISs) of humeral shaft fractures are associated with less radial nerve injury, less soft tissue injury and higher union rate. However, malrotation often occurs in MISs when closed reduction methods are used.

This study aims to define specific palpable landmarks to help surgeons determine the correct torsional angle and reduce the incidence of malrotation.

Methods: Twenty-eight normal humeral computed tomography scans were retrieved from our image database. One line was drawn through the vertices of the intertubercular sulcus of the humeral head in the coronal view, and another line was drawn through the longest axis between the medial and lateral condyles in the coronal view. The angle between these two lines was measured at least 3 times for each scan.
\end{abstract}

Results: The profile of the intertubercular sulcus tangent line of the humeral head and the axis of the distal humerus was identified as the most accurate method for assessing the precision of torsion during MIS for humeral shaft fractures. The transepicondylar axis line is more internally rotated than the intertubercular sulcus tangent line. The mean angle was measured to be 41.1 degrees.

Conclusions: The axis of the distal humeral condyles is internally rotated by approximately 41.1 degrees compared with the intertubercular sulcus tangent line of the humeral head. Minimally invasive surgeries can be performed by using these palpable landmarks. The torsional deformities can be reduced with the proper angle adjustment without the need for fluoroscopy. It can also be used to treat unstable comminuted humeral fractures.

Level of evidence: Retrospective Study, Diagnostic study, Level III.

\section{Background}

Fractures of the humeral shaft are common, as they account for $10 \%$ of long-bone fractures and $3-5 \%$ of all fractures $[1,2]$. Humeral shaft fractures result in a significant burden to society due to lost productivity and wages. In the United States, over 66,000 cases occur annually and account for more than 363,000 days of hospital stays [3]. Both the incidence of humeral shaft fractures and the utilization of surgical interventions have been increasing over time [4]. A bimodal age distribution with one peak was found in men in their thirties, and another peak was found in women in their seventies

\footnotetext{
* Correspondence: u9301013@cmu.edu.tw

${ }^{4}$ Department of Orthopaedics, Shuang Ho Hospital, Taipei Medical University, New Taipei City 23561, Taiwan

${ }^{5}$ Department of Orthopaedics, School of Medicine, College of Medicine,

Taipei Medical University, Taipei City 11031, Taiwan

Full list of author information is available at the end of the article
}

[5]. Among them, the younger patients were included in the high-activity groups. For those with economic responsibility for the household, the recovery of upper limb function is very important.

The goal of treatment for humeral shaft fractures is bony union with an acceptable humeral alignment and a return to the pre-injury level of activity [6]. There is a high risk for pseudoarthrosis, as it occurs in as many as $29 \%$ of cases without surgical treatments $[2,7]$.

Among the many kinds of surgeries that are available for humeral shaft fractures, minimally invasive surgeries (MISs), which were first described by Livani and Belangero [8], are particularly important. MISs have been widely used for reduction and fixation of humeral shaft fractures with good results, as the biology and vascularization of fragments have been maximally preserved. Other advantages include the absence of injury to soft tissue, which leads to maintained blood 
supply to the bone, less blood loss, a shorter operative time [8-13], the best aesthetic result, and a lower rate of complications, such as non-union, radial nerve palsy, and infection [14-16].

The two main minimally invasive surgical techniques for fracture fixation are intramedullary (IM) nailing and minimally invasive plate osteosynthesis (MIPO). Indirect reduction techniques that do not reveal the site of fracture are always used during MISs [17]. Regardless of whether IM nailing or MIPO is performed, a reduction is usually evaluated using fluoroscopy. It is easy to identify valgus or varus deformities, but it is difficult to identify torsional deformities. Torsional deformities of various degrees may occur. Postoperative malrotation exceeding $20^{\circ}$ was found in $40.9 \%$ of a group of patients who underwent MIPO [15]. The degree of malrotation correlates with a decreased range of motion and may be a cause of degenerative arthritis in the long-term [18].

Clinically, torsional alignment requires a surgeon's clinical judgement under intraoperative fluoroscopy as he or she observes the shape of bone fragments or compares the affected humerus with the contralateral side [13]. Not only are patients and staff members in the operating room exposed to radiation, but the operation also takes longer to perform. For pregnant patients, avoiding the use of fluoroscopy during surgery is important. Moreover, for hospitals not equipped with fluoroscopy tools, surgeons may have difficulty identifying humeral deformities in the fracture via the MIS approach.

The angle of humeral head retroversion has been most commonly used for measuring the torsional degrees of the humerus [19-21]. However, when using humeral head retroversion as a torsional reference for the surgical treatment of humeral shaft fractures, fluoroscopic assistance or extensive operative wounds for exposure of the humeral head are usually required.

This study aims to define specific palpable landmarks to help surgeons measure humeral torsion and reduce the incidence of torsional deformities during MIS without fluoroscopic assistance. To the best of our knowledge, this is the first study assessing torsional alignment by examining the specific angle of humeral anatomic torsion using computed tomography (CT) images.

\section{Methods}

\section{Studied population}

All humeral CT scans between February, 2007 and August, 2018 were retrospectively retrieved from the Picture Archiving and Communication System (PACS) at our hospital. The images were selected for analysis according to the following inclusion criteria: (1) age between 18 and 90 years, (2) normal humeral structure without any congenital or acquired deformities, and (3) serial computed tomography records of the whole humerus.

Overall, the studied population comprised 28 paired cases, including 13 women and 15 men with a mean age of 54.9 years (range $18-89$ years, median age 53 years).

This study was approved by the institutional review board (IRB) of Shin Kong Wu Ho-Su Memorial Hospital under IRB number: 20180809R, and was conducted in accordance with the relevant guidelines and regulations.

\section{Determination of reference landmarks}

CT scans of the full-length humerus with a $5-\mathrm{mm}$ slice thickness were performed to study the crosssectional morphology and to identify landmarks for measurement.

The proximal orientation of the humerus was first measured on the section of the deepest intertubercular sulcus. One line (OA line in Fig. 1) was drawn through the vertices of the deepest intertubercular sulcus (points $x$ and $y$ in Fig. 2) of the humeral head in the coronal view. Another line (OB line in Fig. 1) was drawn through the longest axis between the medial and lateral condyle in coronal view. The angle (called the " $\alpha$ " angle in this study) between the tangent line of the intertubercular sulcus and the transepicondylar axis line was identified.

\section{Statistical analysis}

Each measurement was conducted at least three times by the same orthopaedist with 8 years of institutional experience and the average value was used for analysis.

All statistical analyses were performed using IBM SPSS Statistics Subscription version 1.0.0.1174.

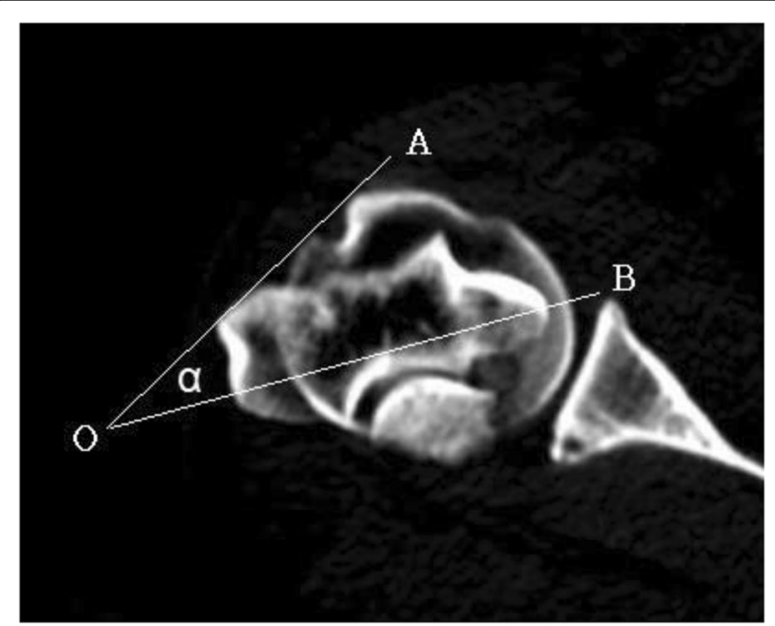

Fig. 1 Two reference axis lines: OA line is the tangent line of the intertubercular sulcus; $\mathrm{OB}$ line is the transepicondylar axis line 


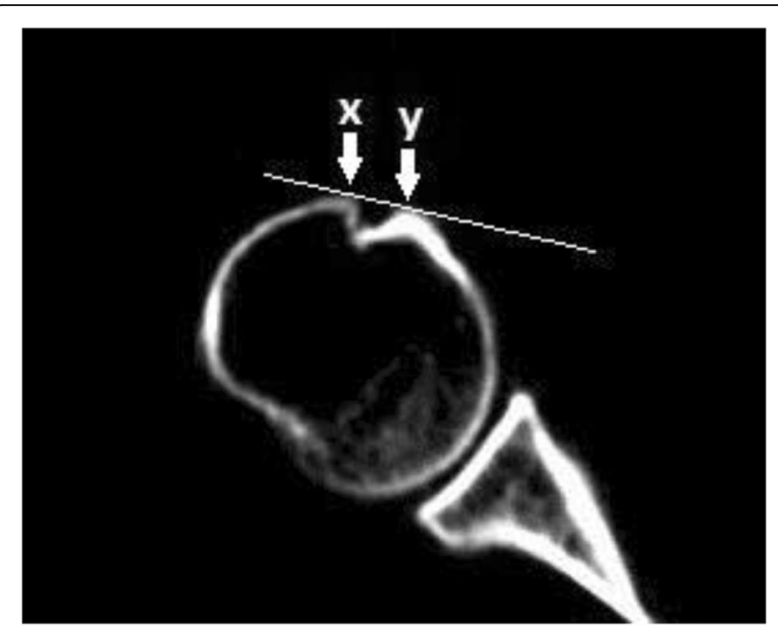

Fig. 2 Points $x$ and $y$ are the vertices of biceps groove. The two points are connected in a tangent line of the deepest intertubercular sulcus

\section{Results}

We collected demographic and measurement data for all 28 subjects (Table 1 ). The " $\alpha$ " angle was correctly identified in all subjects.

The transepicondylar axis line was more internally rotated than the connecting line at the vertices of the intertubercular sulcus. As shown in Table 2, the mean angle was measured to be $41.1^{\circ}$ (range $1.0^{\circ}-73.1^{\circ}$ ), with a standard deviation of $17.1^{\circ}$. The outliers were determined by calculating the $\mathrm{z}$-score, and $\mathrm{z}$-scores greater than 2 or less than -2 were considered outliers. There was only one outlier with an $\alpha$ angle of $1.0^{\circ}$. When the outlier was excluded, the mean angle was $42.6^{\circ}$ (range $10.7^{\circ}-73.1^{\circ}$ ), and the standard deviation was $17.1^{\circ}$ (Table 2).

Table 3 shows the correlations of the $\alpha$ angle with the humeral length and patient age. The correlation coefficient between the $\alpha$ angle and humeral length was 0.42 , while that between the $\alpha$ angle and age was 0.52 .

The intraclass correlation coefficient (ICC) with a twoway random effects model of consistency was used to analyse measurement reliability. For the measurement of the $\alpha$ angle, the intraobserver correlation was excellent (Table 4).

\section{Discussion}

The purpose of this study was to determine the landmarks important for evaluating torsional deviations of the humerus when minimally invasive surgical techniques are used for humeral fracture fixation. The results obtained showed that differences in the angle between the transepicondylar axis line and the connecting line at the vertices of the intertubercular sulcus ranged from $1.0^{\circ}$ to $73.1^{\circ}$, with a mean angle of $41.1^{\circ}$.
Table 1 Demographic and measurement data

\begin{tabular}{|c|c|c|c|}
\hline Patient & Humerus $(\mathrm{cm})$ & a angle $(\circ)$ & z-scores \\
\hline 1 & 26.36 & 55.6 & 0.8 \\
\hline 2 & 28.60 & 59.5 & 1.1 \\
\hline 3 & 28.88 & 23.8 & -1 \\
\hline 4 & 31.74 & 40.9 & 0 \\
\hline 5 & 26.87 & 30.6 & -0.6 \\
\hline 6 & 25.62 & 45.6 & 0.3 \\
\hline 7 & 29.86 & 21.2 & -1.2 \\
\hline 8 & 31.57 & 33.0 & -0.5 \\
\hline 9 & 26.40 & 48.3 & 0.4 \\
\hline 10 & 28.50 & 37.6 & -0.2 \\
\hline 11 & 27.72 & 51.8 & 0.6 \\
\hline 12 & 28.02 & 38.8 & -0.1 \\
\hline 13 & 27.96 & 65.1 & 1.4 \\
\hline 14 & 31.81 & 40.1 & -0.1 \\
\hline 15 & 30.77 & 41.6 & 0 \\
\hline 16 & 32.04 & 60.4 & 1.1 \\
\hline 17 & 28.88 & 10.7 & -1.8 \\
\hline 18 & 27.70 & 38.2 & -0.2 \\
\hline 19 & 31.57 & 73.1 & 1.9 \\
\hline 20 & 31.00 & 1.0 & -2.3 \\
\hline 21 & 30.82 & 25.1 & -0.9 \\
\hline 22 & 28.36 & 37.9 & -0.2 \\
\hline 23 & 30.60 & 26.2 & -0.9 \\
\hline 24 & 29.33 & 48.8 & 0.4 \\
\hline 25 & 31.41 & 65.1 & 1.4 \\
\hline 26 & 28.40 & 31.5 & -0.6 \\
\hline 27 & 32.49 & 64.4 & 1.4 \\
\hline 28 & 28.43 & 34.8 & -0.4 \\
\hline
\end{tabular}

This study identified the profile of the intertubercular sulcus line of the humeral head and the axis of the distal humerus as an accurate tool for assessing the precision of torsion during MISs for humeral shaft fractures without fluoroscopic assistance. The line drawn through the vertices of the intertubercular sulcus line of the humeral head was externally rotated by approximately $41.1^{\circ} \mathrm{com}-$ pared with the axis of the distal humeral condyles.

In practice, surgeons may palpate the bony prominence of the intertubercular sulcus of the proximal humeral fragment as a landmark to evaluate the tangent line. Surgeons may rotate the distal humeral fragment,

Table 2 Clinical validation results on a angle

\begin{tabular}{lllll}
\hline Outlier & Minimum & Maximum & Mean & Standard deviation \\
\hline With & $1.0^{\circ}$ & $73.1^{\circ}$ & $41.1^{\circ}$ & $17.1^{\circ}$ \\
Without & $10.7 \circ$ & $73.1^{\circ}$ & $42.6^{\circ}$ & $15.5^{\circ}$ \\
\hline
\end{tabular}


Table 3 Correlation of a angle with humeral length and age

\begin{tabular}{lll}
\hline Variables & $\begin{array}{l}\text { Pearson product-moment } \\
\text { correlation coefficient }\end{array}$ & $\begin{array}{l}\text { Degree of } \\
\text { correlation }\end{array}$ \\
\hline a angle \& Humeral length & 0.42 & Low \\
a angle \& Age & 0.52 & Moderate \\
\hline
\end{tabular}

making the transepicondylar axis line internally rotated by $41.1^{\circ}$ compared with the tangent line of the intertubercular sulcus. After the fixation is completed and stable, a clinical exam of internal rotation and external rotation of the shoulder joint can be performed to determine whether the forearm is oriented in the proper direction [22]. Thus, the fracture will undergo proper torsional reduction. Additionally, the technique may be effective for unstable comminuted or segmental humeral fractures (AO type 12C) if it is difficult to maintain the fracture in adequate alignment during the operation. A clinical trial should be conducted in the future as a final test. This technique needs to be demonstrated to prevent an increase the numbers of torsional malalignments and complications.

If a preoperative CT scan is available, the fracture can be virtually reduced using 3D system software [23]. The reconstructed model can be used to restore the physiological magnitude of humeral torsion and measure the alpha angle of the humerus.

In this study, the parameters of the humerus were measured using CT. In all 28 cases, the connecting line at the two vertices of the intertubercular sulcus was found to be externally rotated compared with the transepicondylar axis line.

Measurements were made on serial images of the humerus with $5 \mathrm{~mm}$ between each section. Errors may occur because the deepest sulcus and the longest axis of condyles of the sections taken for measurement may not be the actual deepest and longest ones.

Previous research studies considered a humeral malrotation of $15^{\circ}$ in fracture alignment acceptable [24]. Although the standard deviation of the $\alpha$ angle obtained in this study was $17.1^{\circ}$, the mean angle may still be used as a reference for reduction. Statistically, an extreme outlier in the present data was identified. The standard deviation without the outlier was $15.5^{\circ}$, which is close to the abovementioned acceptable degree $\left(15^{\circ}\right)$ of humeral malrotation.

The proximal incision during MIPO is made with the deltopectoral approach [25]. The surgeon can directly

Table 4 Intraclass correlation coefficients (ICC) in the intraobserver measurement

\begin{tabular}{lll}
\hline Intraobserver reliability & Single measures & Average measures \\
\hline ICC & 0.974 & 0.991 \\
\hline
\end{tabular}

touch the biceps sulcus as a landmark. The soft tissue around the elbow is thin in most cases. Epicondyles can be easily touched to identify the transepicondylar axis. The axis identified by the surgeon is not very different from the actual direction.

The palpable proximal and distal osseous landmarks (intertubercular sulcus, medial and lateral epicondyles) located by orthopaedic surgeons are slightly different from the imaging landmarks. For example, the biceps groove is located at the proximal humerus and becomes shallower toward the inferior end. The proximal landmark over the intertubercular sulcus in this study was obtained from the deepest site. Surgeons may not be able to locate the deepest point of the bicipital groove. However, extensive surgical experience and good judgement can increase the accuracy of identifying this groove. Highly trained and specialized orthopaedic surgeons can precisely locate the deepest groove and other anatomical landmarks.

However, the landmarks are not applicable in some situations, such as when the humeral head or the distal humerus is severely deformed due to acquired or congenital disorders.

In past research, a strong relationship has been shown to exist between humeral torsion variables obtained with ultrasound and CT [26]. If ultrasound equipment is available in the operating room, an ultrasonographic assessment of the humeral retroversion method can be used as a secondary confirmation [22]. In addition, when the patient's soft tissue layer is thick and it is difficult to palpate the bony landmarks, ultrasonic positioning can be used to identify the transepicondylar axis.

Various techniques have been employed to measure torsional parameters of the humeral bone. Retroversion of the humeral head is most commonly used for defining the angular difference between the orientation of the proximal humeral head and the axis of the elbow at the distal humerus [19]. However, the results are highly variable, ranging in some case series from $-6^{\circ}$ to $50^{\circ}$ [27-30].

Nevertheless, obtaining the retroversion angle in operation requires fluoroscopic assistance, and it is difficult to confirm whether the proximal line is perpendicular to the articular surface.

A previous study used the bicipital groove of the humeral head to predict the torsional state of the humerus for intraoperative evaluations, but fluoroscopy was still needed with this approach [31].

To the best of our knowledge, no other study has used $\mathrm{CT}$ to measure humeral torsion with the tangent line of the intertubercular sulcus. A similar study measured humeral head retroversion with lateralization of the intertubercular groove using CT [32], which seems to be valuable for anatomical imaging but unsuitable for clinical orthopaedic surgeries. 
This study also identified the correlations of the $\alpha$ angle with the humeral length and patient age. The correlation between the $\alpha$ angle and humeral length was statistically low, while that between the $\alpha$ angle and patient age was moderate. In the absence of a strong correlation with the $\alpha$ angle, age and humeral length need not be considered in clinical-surgical evaluations.

There were 13 right and 15 left humerus bones included in the research study. The mean alpha angle in the present study was $37.4 \pm 18.6$ degrees on the right side and $44.3 \pm 15.7$ degrees on the left side. The sample size is small, so the statistical significance of the results is unclear.

Previous studies have shown that the dominant arm of patients has a higher retroversion angle than the contralateral arm. On average, the degree of retroversion is 10.6 degrees larger in the dominant arm compared with the nondominant arm in overhead throwing athletes [33, 34]. Although the alpha angle is not an exact measure of humeral head retroversion, perhaps the angles on the bilateral humerus are different for people who have participated in throwing sports. However, whether the participants in our study practised throwing sports was not recorded. In a future study, we can add this factor to determine whether it has statistical significance.

Finally, only 28 extremities from 28 participants were included in the analysis. The volume of data in our imaging system limited our sample size. While more patients should be included in future prospective research studies, the costs and radiation exposure associated with $\mathrm{CT}$ scans should be taken into consideration when designing these studies.

\section{Conclusions}

The intertubercular sulcus and humeral condyles are easy to identify by palpation and are useful landmarks. Compared with the tangent line of the intertubercular sulcus, the transcondylar axis is internally rotated by 41.1 degrees. The alpha angle can be effectively used in minimally invasive surgeries or unstable comminuted fractures to reduce torsional malalignment without fluoroscopic assistance. However, additional clinical studies are necessary to further verify these conclusions.

\section{Abbreviations}

AO: Arbeitsgemeinschaft für Osteosynthesefragen (German for "Association for the Study of Internal Fixation"); CT: Computed tomography; ICC: Intraclass correlation coefficients (ICC); IM: Intramedullary; IRB: Institutional review board; MIPO: Minimal invasive plate osteosynthesis; MIS: Minimally invasive surgeries; ORIF: Open reduction internal fixation; PACS: Picture Archiving and Communication System

\section{Acknowledgements}

We are immensely grateful to Carl Chen and Juny Chu for their comments on an earlier version of the manuscript.

\section{Authors' contributions}

YLC was the primary researcher involved with study conception, methodology and data analysis. CKC was responsible for data curation. YCL and TWL performed writing-review and editing. CKL oversaw the project, and with YLC, were responsible for drafting the manuscript, interpretation of data, and manuscript writing. All authors read and approved the final manuscript.

\section{Funding}

This research received no external funding.

\section{Availability of data and materials}

The datasets used and analysed during the current study are available from the corresponding author on reasonable request.

\section{Ethics approval and consent to participate}

Our study was approved by the institutional review board (IRB) of Pusan Shin Kong Wu Ho-Su Memorial Hospital (decision number, 20180809R). The ethics committee that approved the study ruled that no formal consent was necessary.

Consent for publication

Not applicable.

\section{Competing interests}

The authors declare that they have no competing interests.

\section{Author details}

${ }^{1}$ Institute of Biomedical Engineering, National Taiwan University, Taipei 100 Taiwan. ${ }^{2}$ Department of Orthopaedics, Taipei Municipal Wanfang Hospital, Taipei 11696, Taiwan. ${ }^{3}$ Department of Orthopedic Surgery, Shin Kong Wu Ho-Su Memorial Hospital, Taipei 11101, Taiwan. ${ }^{4}$ Department of

Orthopaedics, Shuang Ho Hospital, Taipei Medical University, New Taipei City 23561, Taiwan. ${ }^{5}$ Department of Orthopaedics, School of Medicine, College of Medicine, Taipei Medical University, Taipei City 11031, Taiwan. ${ }^{6}$ Graduate Institute of Biomedical Optomechatronics, College of Biomedical Engineering Research Center of Biomedical Device, Taipei Medical University, Taipei City 11301, Taiwan.

Received: 23 August 2019 Accepted: 5 February 2020

Published online: 10 February 2020

References

1. Sarmiento A, Zagorski JB, Zych GA, Latta LL, Capps CA. Functional bracing for the treatment of fractures of the humeral diaphysis. JBJS. 2000;82:478-86

2. Walker M, Palumbo B, Badman B, Brooks J, Gelderen JV, Mighell M. Humeral shaft fractures: a review. J Shoulder Elb Surg. 2011;20:833-44.

3. Hoang-Kim A, Goldhahn J, Hak DJ. Humeral shaft fractures. Evidence-Based Orthopedics. 2011. p. 366-373. https://doi.org/10.1002/9781444345100.ch42. ISBN-13:978-1-4051-8476-2.

4. Schoch BS, Padegimas EM, Maltenfort M, Krieg J, Namdari S. Humeral shaft fractures: national trends in management. J Orthop Traumatol. 2017;18:259-63.

5. Tytherleigh-Strong G, Walls N, McQueen MM. The epidemiology of humeral shaft fractures. J Bone Joint Surg Br Vol. 1998;80:249-53.

6. Kumar BS, Soraganvi P, Satyarup D. Treatment of middle third humeral shaft fractures with anteromedial plate osteosynthesis through an anterolateral approach. Malays Orthop J. 2016;10:38.

7. Rutgers $M$, Ring $D$. Treatment of diaphyseal fractures of the humerus using a functional brace. J Orthop Trauma. 2006;20:597-601.

8. Livani B, Belangero WD. Osteossíntese de fratura diafisária do úmero com placa em ponte: apresentação e descrição da técnica. Acta Ortopédica Brasileira. 2004. https://doi.org/10.1590/S1413-78522004000200007.

9. Apivatthakakul T, Arpornchayanon O, Bavornratanavech S. Minimally invasive plate osteosynthesis (MIPO) of the humeral shaft fracture: is it possible? A cadaveric study and preliminary report. Injury. 2005;36:530-8.

10. Concha JM, Sandoval A, Streubel PN. Minimally invasive plate osteosynthesis for humeral shaft fractures: are results reproducible? Int Orthop. 2010;34:1297-305. 
11. Ji F, Tong D, Tang H, Cai X, Zhang Q, Li J, et al. Minimally invasive percutaneous plate osteosynthesis (MIPPO) technique applied in the treatment of humeral shaft distal fractures through a lateral approach. Int Orthop. 2009;33:543-7.

12. Rüedi TP, Murphy WM. AO principles of fracture management. Davos: AO Publishing \& Stuttgart New York: Georg Thieme Verlag; 2000.

13. Shetty MS, Kumar MA, Sujay KT, Kini AR, Kanthi KG. Minimally invasive plate osteosynthesis for humerus diaphyseal fractures. Indian J Orthop. 2011;45:520

14. Esmailiejah AA, Abbasian MR, Safdari F, Ashoori K. Treatment of humeral shaft fractures: minimally invasive plate osteosynthesis versus open reduction and internal fixation. Trauma Monthly. 2015;20. http://scholar. google.com/scholar_lookup?\&title=Treatment $\% 20$ of\%20humeral\%20shaft $\% 2$ ofractures\%3A\%20minimally\%20invasive\%20plate\%20osteosynthesis\%2 Oversus\%20open\%20reduction\%20and\%20internal\%20fixation\&journal= Trauma\%20Mon\&volume=20\&issue=3\&publication_year=2015\&author= Esmailie.

15. Wang C, Li J, Li Y, Dai G, Wang M. Is minimally invasive plating osteosynthesis for humeral shaft fracture advantageous compared with the conventional open technique? J Shoulder Elb Surg. 2015;24:1741-8.

16. Zhao J-G, Wang J, Meng X-H, Zeng X-T, Kan S-L. Surgical interventions to treat humerus shaft fractures: a network meta-analysis of randomized controlled trials. PLoS One. 2017;12:e0173634.

17. Lee H-J, Oh C-W, Oh J-K, Apivatthakakul T, Kim J-W, Yoon J-P, et al. Minimally invasive plate osteosynthesis for humeral shaft fracture: a reproducible technique with the assistance of an external fixator. Arch Orthop Trauma Surg. 2013;133:649-57.

18. Li Y, Wang C, Wang M, Huang L, Huang Q. Postoperative malrotation of humeral shaft fracture after plating compared with intramedullary nailing. J Shoulder Elb Surg. 2011;20:947-54.

19. Boileau P, Bicknell RT, Mazzoleni N, Walch G, Urien JP. CT scan method accurately assesses humeral head retroversion. Clin Orthop Relat Res. 2008;466:661-9.

20. Guenoun D, Le Corroller T, Lagier A, Pauly V, Champsaur P. Correlation between the retroversion of the humeral head and the orientation of the intertubercular sulcus: a CT scan anatomical study. Surg Radiol Anat. 2015;37:357-61.

21. Hernigou P, Duparc F, Hernigou A. Determining humeral retroversion with computed tomography. JBJS. 2003;85:1163.

22. Hawi N, Razaeian S, Krettek C, Meller R, Liodakis E. Torsional malalignment of the humerus. Unfallchirurg. 2018;121:199-205.

23. Omar M, Zeller A-N, Gellrich N-C, Rana M, Krettek C, Liodakis E. Application of a customized 3D printed reduction aid after external fixation of the femur and tibia: technical note. Int J Med Robotics Comput Assist Surg. 2017;13:e1803.

24. Klenerman L. Fractures of the shaft of the humerus. J Bone Joint Surg Br Vol. 1966;48-B:105-11.

25. Tan JCH, Kagda FHY, Murphy D, Thambiah JS, Khong KS. Minimally invasive helical plating for shaft of Humerus fractures: technique and outcome. Open Orthop J. 2012;6:184-8.

26. Myers JB, Oyama S, Clarke JP. Ultrasonographic assessment of humeral Retrotorsion in baseball players: a validation study. Am J Sports Med. 2012;40:1155-60.

27. Boileau $\mathrm{P}$, Walch $\mathrm{G}$. The three-dimensional geometry of the proximal humerus: implications for surgical technique and prosthetic design. J Bone Joint Surg Br Vol. 1997;79:857-65.

28. Evans FG, Krahl VE. The torsion of the humerus: a phylogenetic survey from fish to man. Am J Anatomy. 1945;76:303-37.

29. Krahl VE. The torsion of the humerus: its localization, cause and duration in man. Am J Anatomy. 1947;80:275-319.

30. Robertson DD, Yuan JIE, Bigliani LU, Flatow EL, Yamaguchi K. Threedimensional analysis of the proximal part of the humerus: relevance to arthroplasty. JBJS. 2000;82:1594-602.

31. Park S-J, Kim E, Jeong HJ, Lee J, Park S. Prediction of the rotational state of the humerus by comparing the contour of the contralateral bicipital groove: method for intraoperative evaluation. Indian J Orthop. 2012;46:675.

32. Cassagnaud X, Maynou C, Petroff E, Dujardin C, Mestdagh H. A study of reproducibility of an original method of $C T$ measurement of the lateralization of the intertubercular groove and humeral retroversion. Surg Radiol Anat. 2003;25:145-51.
33. Reagan KM, Meister K, Horodyski MB, Werner DW, Carruthers C, Wilk K Humeral retroversion and its relationship to Glenohumeral rotation in the shoulder of college baseball players. Am J Sports Med. 2002;30:354-60.

34. Chant CB, Litchfield R, Griffin S, Thain LMF. Humeral head retroversion in competitive baseball players and its relationship to Glenohumeral rotation range of motion. J Orthop Sports Phys Ther. 2007;37:514-20.

\section{Publisher's Note}

Springer Nature remains neutral with regard to jurisdictional claims in published maps and institutional affiliations.
Ready to submit your research? Choose BMC and benefit from:

- fast, convenient online submission

- thorough peer review by experienced researchers in your field

- rapid publication on acceptance

- support for research data, including large and complex data types

- gold Open Access which fosters wider collaboration and increased citations

- maximum visibility for your research: over $100 \mathrm{M}$ website views per year

At BMC, research is always in progress.

Learn more biomedcentral.com/submissions 